\title{
EFEITO DA RAZÃO R ENTRE TENSÕES NA RESISTÊNCIA AO CRESCIMENTO DE TRINCA POR FADIGA DE CINCO AÇOS DE APLICAÇÃO EM TRILHO FERROVIÁRIO*
}

Tamara Caroline Guimarães Vilela ${ }^{1}$ Leonardo Barbosa Godefroid² Luiz Cláudio Cândido² Geraldo Lúcio de Faria ${ }^{3}$

\section{Resumo}

Neste trabalho foi avaliado o comportamento de propagação de trinca por fadiga de cinco aços utilizados na fabricação de trilhos empregados na construção e manutenção de vias permanentes brasileiras, por meio da realização de ensaios de propagação de trinca por fadiga (da/dN $x \Delta K)$, utilizando-se a razão de tensões de carregamento $R=0,7$ e comparação com os resultados obtidos com $R=0,1$. Vários modelos de previsão do comportamento em fadiga foram avaliados. Observou-se a influência de $R$ nos valores mais próximos ao limiar $\Delta \mathrm{K}_{\mathrm{TH}}$, com diminuição considerável no valor de $\Delta \mathrm{K}_{\mathrm{TH}}$ para $\mathrm{R}=0,7$. Dentre os modelos matemáticos usados no ajuste das curvas sigmoidais $d a / d N \times \Delta K$, o que forneceu melhor ajuste foi 0 ajuste de Hartman e Schijve. O parâmetro $K^{*}$ proposto por Kujawski reduziu os efeitos de $R$ na tentativa de unificar os dados em uma única e estreita banda de dispersão, porém, na Região I da curva, os dados ainda ficaram bastante dispersos.

Palavras-chave: Resistência ao crescimento de trinca por fadiga; Razão R; Modelos de ajuste; Trilho ferroviário.

\section{EFFECT OF LOAD RATIO, R, ON FATIGUE CRACK GROWTH RESISTANCE OF FIVE STEELS FOR RAILROAD APPLICATION}

\section{Abstract}

This study evaluated the fatigue crack propagation of five steels used in the manufacture of rails used in the construction and maintenance of Brazilian railroads, conducting fatigue crack propagation tests $(d a / d N \times \Delta K)$ with load ratio $\mathrm{R}=0.7$ and comparing with results obtained with $R=0.1$. Many models are used to evaluate the fatigue behavior of the steels. It is noticed the influence of $R$ closer to $\Delta K_{T H}$ values, with considerable decrease in $\Delta \mathrm{K}_{T H}$ value for $\mathrm{R}=0.7$. Among the mathematical models used in the setting of sigmoidal curves $d a / d N \times \Delta K$, the setting of Hartman and Schijve provided the best fit. The $K^{*}$ parameter proposed by Kujawski reduced the effects of $R$ in an attempt to unify the data into single, narrow band dispersion; however in Region I of the curve the data were still widespread.

Keywords: Fatigue crack growth resistance; R-ratio; Fitting models; Railroad.

1 Engenheira Metalúrgica, Mestranda em Engenharia de Materiais, REDEMAT, UFOP, Ouro Preto, Minas Gerais, Brasil.

2 Engenheiro Metalúrgico, Dr., Professor, Departamento de Engenharia Metalúrgica e de Materiais (DEMET), UFOP, Ouro Preto, Minas Gerais, Brasil.

3 Físico, Dr. Professor, Departamento de Engenharia Metalúrgica e de Materiais (DEMET), UFOP, Ouro Preto, Minas Gerais, Brasil. 


\section{INTRODUÇÃO}

Dentre os meios de transporte utilizados para a movimentação de grandes volumes de carga, o transporte ferroviário é um dos mais econômicos e eficazes e, no Brasil, apesar do transporte rodoviário ser mais utilizado, a ferrovia se destaca no transporte de cargas de alto fluxo de produção tais como as de minério de ferro, metais e grãos [1, 2].

Ao longo da malha ferroviária brasileira, podem ser encontrados trilhos com características diferentes, de diversos fabricantes e com composições químicas distintas, o que faz com que cada trilho tenha um desempenho diferente, tornando necessário realizar uma análise das características microestruturais, das propriedades mecânicas básicas e do comportamento em fadiga desses componentes para melhor utilização, inspeção e manutenção dos trilhos [3].

Como muitos componentes metálicos, os trilhos estão submetidos ao carregamento cíclico, o que pode levar a uma falha por fadiga, parcial ou completa. O contínuo aumento de cargas por eixo, da frequência de trens e da velocidade de circulação causam um aumento correspondente de tensões e desgaste na via ao longo do tempo, o que pode levar ao aparecimento de defeitos [4,5]. Dentre os vários tipos de defeitos que podem ocorrer nos trilhos, existe um, denominado trinca transversal por fadiga (detail fracture), que é o mais comumente encontrado em linhas continuamente soldadas e que pode provocar a separação total do segmento de trilho [1]. Este tipo de falha por fadiga pode levar ao descarrilamento catastrófico da composição, tendo consequências como grandes perdas econômicas, graves prejuízos ambientais, perda da confiança do público e até perdas humanas $[2,5,6]$. Neste trabalho, utilizando os conceitos da Mecânica de Fratura, foi avaliado o comportamento de propagação de trinca por fadiga de cinco aços para trilhos empregados na construção e manutenção da via permanente brasileira, por meio de ensaios de propagação de trinca por fadiga $(d a / d N \times \Delta K)$, utilizando-se a razão de tensões de carregamento $R=0,7$ e comparação com os resultados obtidos para $R=$ 0,1 . Alguns modelos empíricos foram aplicados para previsão do crescimento de trinca por fadiga. Trata-se de um trabalho inserido no projeto de cooperação GESFRAM/UFOP-VLI/FCA/VALE, onde já foram caracterizados, para os cinco aços, a tenacidade à fratura e 0 crescimento de trinca por fadiga para uma razão de tensões $R=0,1[3,7]$.

\section{MATERIAIS E MÉTODOS}

Os cinco aços estudados derivam de segmentos de cinco trilhos diferentes produzidos por distintos fabricantes de procedência estrangeira. De acordo com os fabricantes, o trilho $\mathrm{S}$ se enquadra na especificação de trilho do tipo standard e os demais trilhos são classificado como trilho premium. A Tabela 1 apresenta a composição química dos cinco aços utilizados neste trabalho.

Tabela 1. Composição química de cinco aços de trilhos ferroviários (\% em massa)

\begin{tabular}{cccccccccc}
\hline Trilho & $\mathbf{C}$ & $\mathbf{M n}$ & $\mathbf{S i}$ & $\mathbf{P}$ & $\mathbf{S}$ & $\mathbf{C r}$ & $\mathbf{N i}$ & Mo & $\mathbf{V}$ \\
\hline S & 0,731 & 1,24 & 0,54 & 0,0151 & 0,0047 & 0,218 & 0,0259 & 0,0166 & 0,0032 \\
\hline P1 & 0,792 & 0,88 & 0,30 & 0,0173 & 0,0043 & 0,209 & 0,0227 & 0,0166 & 0,0016 \\
\hline P2 & 0,836 & 0,91 & 0,26 & 0,0190 & 0,0091 & 0,218 & 0,0130 & - & 0,0010 \\
\hline P3 & 0,763 & 1,02 & 0,22 & 0,0160 & 0,0098 & 0,211 & 0,0180 & - & 0,0010 \\
\hline P4 & 0,754 & 0,81 & 0,69 & 0,0160 & 0,0068 & 0,319 & 0,0380 & 0,0010 & 0,0870 \\
\hline
\end{tabular}


Pode-se observar que o aço $S$ apresenta o menor teor de carbono entre os cinco aços estudados. Observa-se também que os aços apresentam teores distintos de $\mathrm{Mn}$ e $\mathrm{Si}$, que naturalmente vão alterar a temperabilidade do material. Os aços apresentam também diferentes teores de elementos de liga, o que vai certamente mudar as suas propriedades mecânicas.

Os ensaios de propagação de trinca por fadiga foram realizados de acordo com a Norma ASTM 647/08 [8], em uma máquina servo-hidráulica MTS de 10 toneladas, na frequência de $30 \mathrm{~Hz}$, à temperatura ambiente e com razão $\mathrm{R}$ entre tensões de 0,7 . Curvas tamanho de trinca em função do número de ciclos foram obtidas e transformadas em curvas taxa de propagação de trinca $(d a / d N)$ em função do fator cíclico de intensidade de tensões $(\Delta K)$. Foram utilizados dez corpos de prova do tipo dobramento $\mathrm{SE}(\mathrm{B})$, conforme ilustrado na Figura 1, sendo dois corpos de prova para cada tipo de aço.

(a)
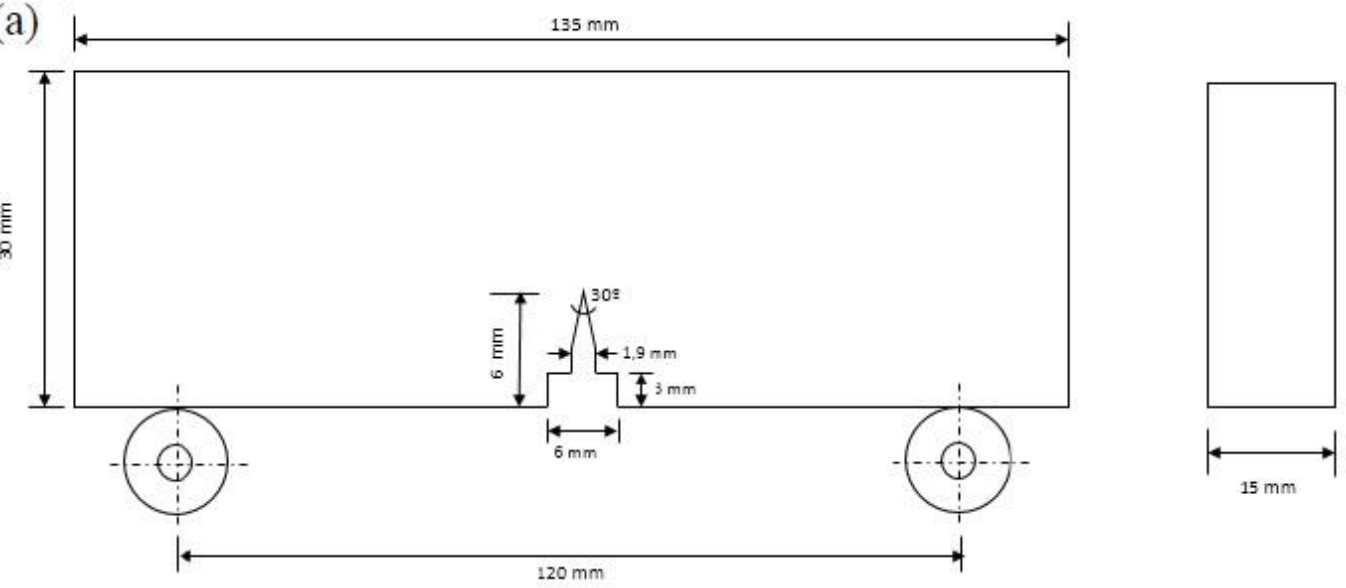

(b)

\begin{tabular}{|c|c|c|}
\hline Espessura & B (mm) & 15 \\
\hline Altura & $\mathbf{W}(\mathbf{m m})$ & 30 \\
\hline Distância entre apoios & $\mathbf{S}(\mathbf{m m})$ & 120 \\
\hline Pré-trinca & $\mathbf{a}(\mathbf{m m})$ & 9 \\
\hline
\end{tabular}

Figura 1. (a) Representação esquemática o corpo de prova para ensaio de propagação de trinca por fadiga. (b) Dimensões dos corpos de prova segundo a Norma ASTM E647/08. Adaptado de [8].

A retirada dos corpos de prova foi realizada na região do boleto, à $2 \mathrm{~mm}$ da superfície de rolamento, segundo orientação L-S, conforme ilustrado na Figura 2, de tal forma que a direção de propagação da trinca no corpo de prova fosse a mesma que ocorre no boleto do trilho em serviço (direção transversal). Nesta configuração, a ponta da trinca situa-se numa região de microestrutura mais homogênea e de dureza mais baixa, representando trincas de origem interna.
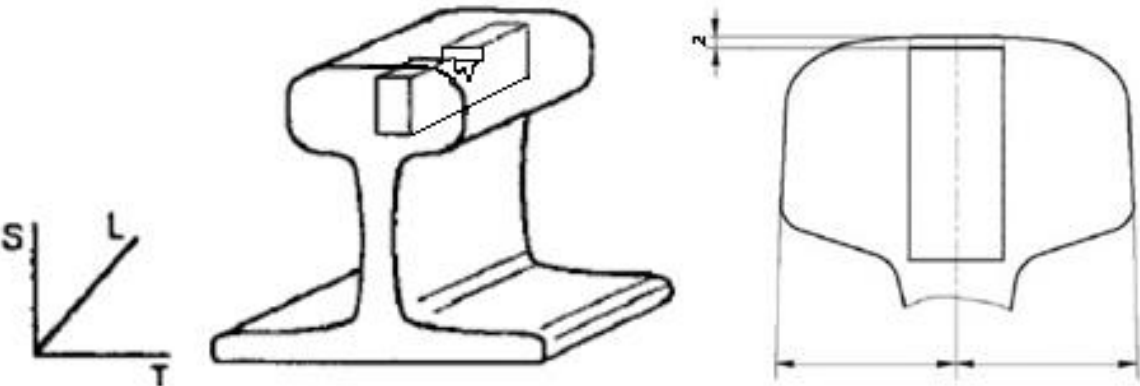

Figura 2. Posição de retirada dos corpos de prova para ensaio de propagação de trinca por fadiga [3]. 
As superfícies de fratura foram analisadas em um microscópio eletrônico de varredura (MEV) TESCAN Vega3.

Com o auxílio do software OriginPro 9.0, alguns modelos empíricos foram aplicados para previsão do crescimento de trinca por fadiga. As Equações 1-6 referem-se a ajustes da curva sigmoidal $d a / d N \times \Delta K$

- Equação de Paris [9]:

- Equação de Collipriest et al. [10]:

$$
\frac{d a}{d N}=C(\Delta K)^{m}
$$

$$
\frac{d a}{d N}=C\left(K_{C} \Delta K_{T H}\right)^{m / 2} \exp \left[\ln \left(\frac{K_{C}}{\Delta K_{T H}}\right)^{m / 2} \times \tanh ^{-1} \frac{\ln \left(\Delta K^{2} / \Delta K_{T H} K_{C}(1-R)\right)}{\ln \left(K_{C}(1-R) / \Delta K_{T H}\right)}\right]
$$

- Equação de NASGRO [11]:

- Equação de Priddle [12]:

$$
\frac{d a}{d N}=\frac{C \Delta K^{m}\left(1-\left(\Delta K_{T H} / \Delta K\right)\right)^{p}}{\left(1-\left(\Delta K / K_{C}(1-R)\right)\right)^{q}}
$$

- Equação de Forman et al. [13]

$$
\frac{d a}{d N}=C\left(\frac{\Delta K-\Delta K_{T H}}{K_{C}-(\Delta K /(1-R))}\right)^{m}
$$

- Equação de Hartman e Schijve [14]:

$$
\frac{d a}{d N}=\frac{C(\Delta K)^{m}}{\left((1-R) K_{c}-\Delta K\right)}
$$

onde:

$$
\frac{d a}{d N}=\frac{D\left(\Delta K-\Delta K_{T H}\right)^{\lambda}}{\left((1-R) K_{C}-\Delta K\right)}
$$

$d a / d N$ : taxa de crescimento de trinca por fadiga

$\Delta K$ : força motriz para crescimento de trinca por fadiga

$\Delta K_{T H:}$ limiar de força motriz

$K_{C}$ : tenacidade à fratura

$R$ : relação entre tensões na fadiga

$C, D, \lambda, m, n, p, q$ : constantes de ajustes

A Equação 7 apresenta um parâmetro de força motriz, K*, proposto por Kujawski [15], que não invoca dados de fechamento de trinca, mas consolida os dados para diferentes razões $\mathrm{R}$ em uma única banda de dispersão. Isto é, este parâmetro tenta unificar as curvas da taxa de crescimento de trinca por fadiga para diferentes razões $R$, minimizando os efeitos de $R$.

$$
K^{*}=\left(K_{\text {máx }}\right)^{\alpha}\left(\Delta K^{+}\right)^{1-\alpha}
$$

onde $\alpha$ é um parâmetro de correlação e $\Delta K^{+}=\Delta K$ para $R \geq 0$. Considerando duas curvas $d a / d N$ obtidas para $R_{2} \geq R_{1} \geq 0$, qualquer valor arbitrário de $d a / d N=$ constante resultará no mesmo parâmetro de força motriz, $K^{K}$. Então, rearranjando a Equação 7 , chega-se à Equação 8.

$$
\alpha=\frac{\log \left(\Delta K_{1}^{+} / \Delta K_{2}^{+}\right)}{\log \left(1-R_{1} / 1-R_{2}\right)}
$$

Deve-se, então, fazer um gráfico com os valores de $\alpha$ versus log $d a / d N$, obtendo-se assim um valor médio para $\alpha$. Quando este a médio é determinado, a Equação 7 pode ser usada para reunir os dados de crescimento de trinca por fadiga com diferentes razões $\mathrm{R}$ em uma curva "mestre" ou faixa de dispersão [15]. 


\section{RESULTADOS E DISCUSSÃO}

As curvas sigmoidais $d a / d N \times \Delta K$ obtidas nos ensaios de fadiga, para $\mathrm{R}=0,1 \mathrm{e}$ $R=0,7$, são apresentadas na Figura 3. Percebe-se o efeito deletério do aumento da razão $\mathrm{R}$ no comportamento de todos os aços estudados, o que está de acordo com a literatura [16-19]. Posto isto, deve-se comentar que a forte sensibilidade à razão $R$ entre tensões para os aços estudados indica o risco de ocorrência de uma fratura catastrófica, caso ocorra uma variação de carregamento na linha ferroviária.

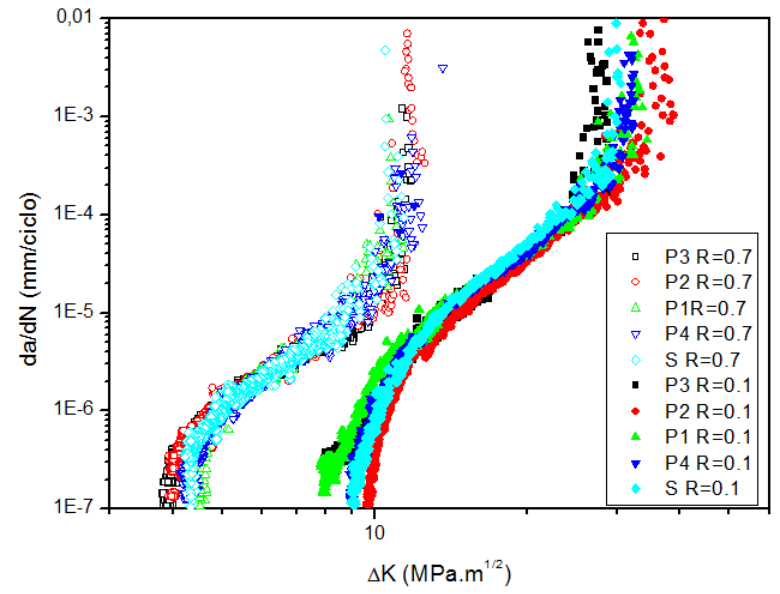

Figura 3. Curvas sigmoidais, em escala bi-logarítmica, de propagação de trinca por fadiga dos cinco aços estudados, efeito da razão $R$ entre tensões.

A diminuição do valor de $\Delta \mathrm{K}_{\mathrm{TH}}$ que acompanhou o aumento do valor de $\mathrm{R}$ é consistente com a redução dos níveis de fechamento de trinca [20, 21]. A Figura 4 revela o desvio de linearidade da curva $P$ x COD, indicando em (a) a presença de fechamento de trinca para $R=0,1$ e em (b) a ausência de fechamento para $R=0,7$.

(a)

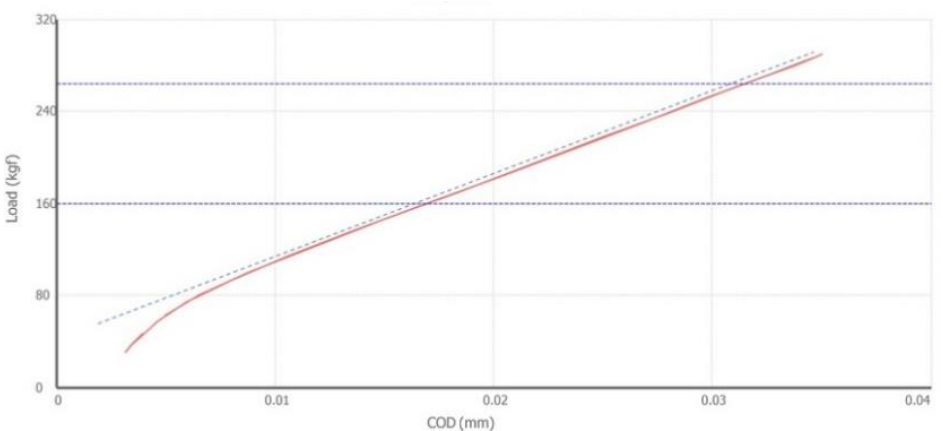

(b)

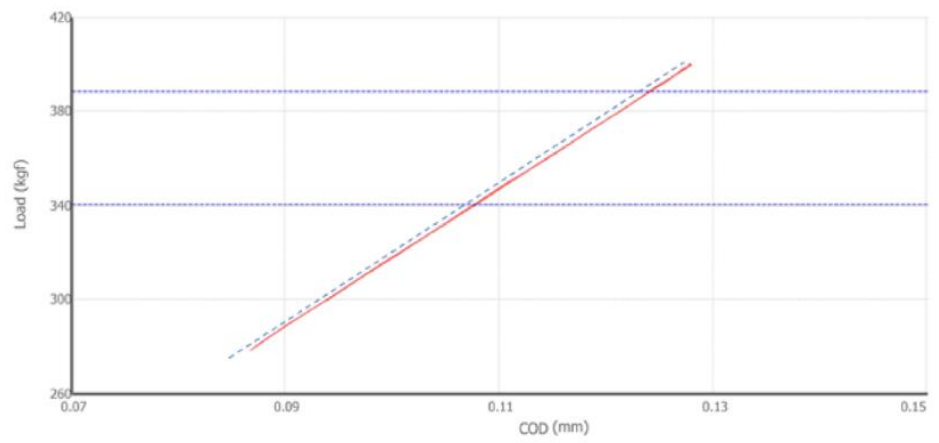

Figura 4 - Curva carga x COD (em vermelho) para um dos aços estudados. A reta tracejada em azul foi construída para realçar o fechamento de trinca na situação (a) e sua ausência na situação (b). 
Para explicitar o efeito de $\mathrm{R}$ no desempenho dos aços, a Figura 5 mostra a dependência do valor de $\Delta \mathrm{K}_{\mathrm{TH}}$ (limiar de força motriz) com este parâmetro.

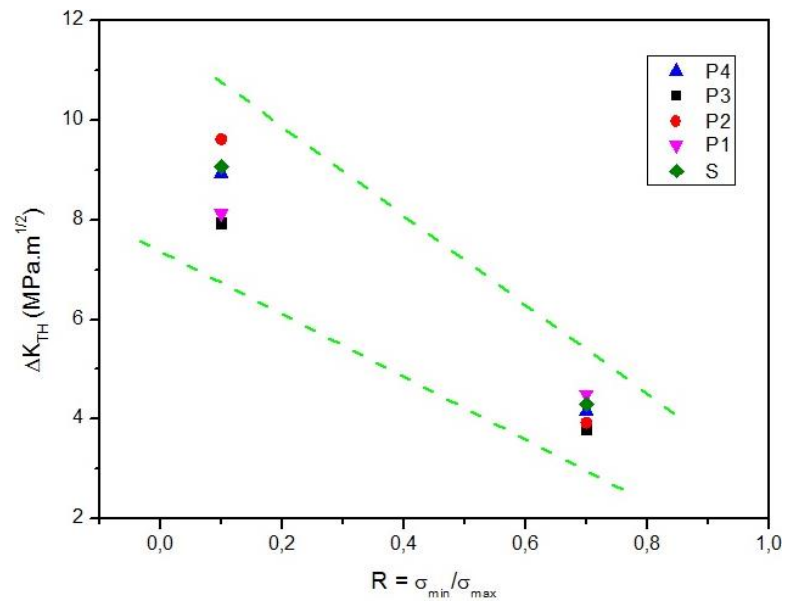

Figura 5. Efeito da razão $R$ entre tensões no limiar $\Delta K_{T H}$ para os cinco aços estudados.

A Tabela 2 apresenta os valores de $\Delta \mathrm{K}_{\mathrm{TH}}$ encontrados nos ensaios, mostrando que há pouca diferença, entre os cinco aços, nos valores de $\Delta \mathrm{K}_{\mathrm{TH}}$, tanto para $\mathrm{R}=0,1$ quanto para $R=0,7$. Isto se deve ao fato de que a composição dos cinco aços é bastante parecida entre si, não resultando em valores de propriedades muito distintos.

Tabela 2. Valores de $\Delta K_{T H}$ (MPa.Vm) quando $R=0,1$ e $R=0,7$ para os cinco aços estudados

\begin{tabular}{c|c|c}
\hline Aço & $\Delta \mathbf{K}_{\mathbf{T H}}-\mathbf{R}=\mathbf{0 , 1}$ & $\Delta \mathbf{K}_{\mathbf{T H}}-\mathbf{R}=\mathbf{0 , 7}$ \\
\hline S & 9,1 & 4,3 \\
\hline P1 & 8,1 & 4,5 \\
\hline P2 & 9,8 & 3,9 \\
\hline P3 & 8,1 & 3,8 \\
\hline P4 & 9,1 & 4,2 \\
\hline
\end{tabular}

A Figura 6 apresenta o modelamento matemático realizado nas curvas sigmoidais $d a / d N \times \Delta K$ com $R=0,7$ e $R=0,1$ para todos os cinco aços, onde foram adotadas as Equações 1 - 6, apresentadas no item 2.
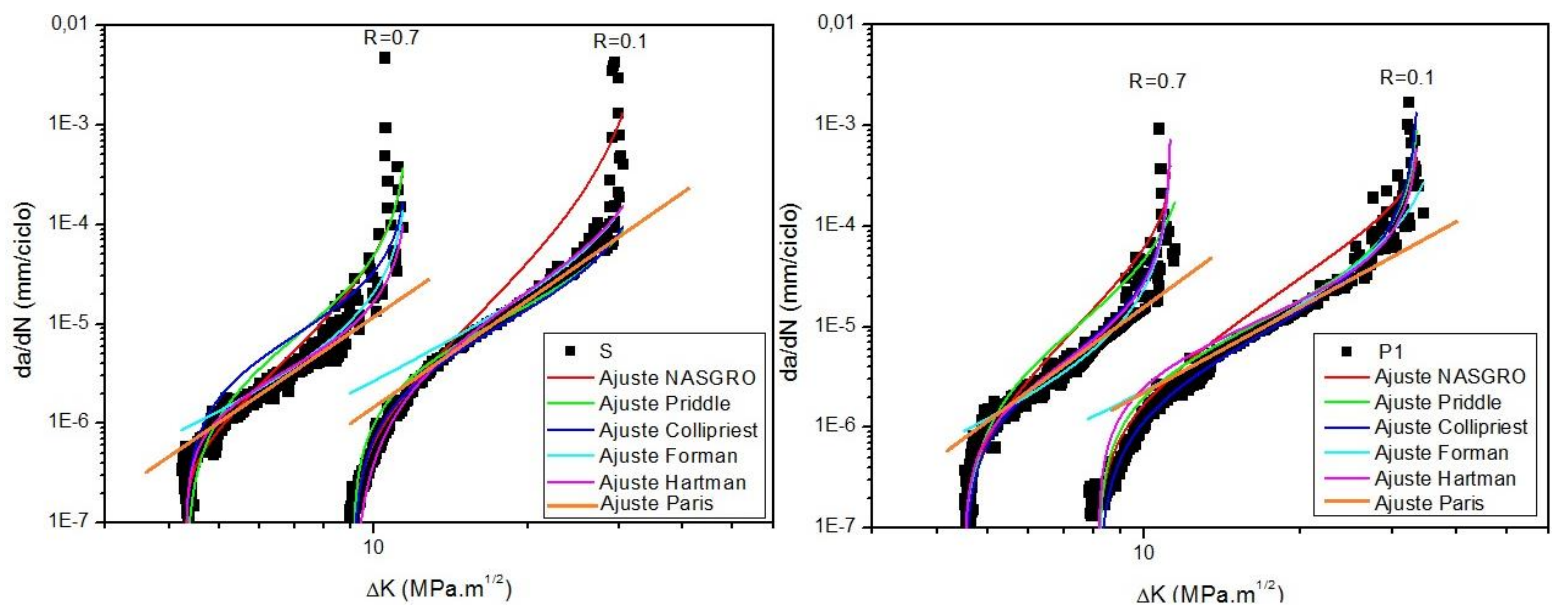

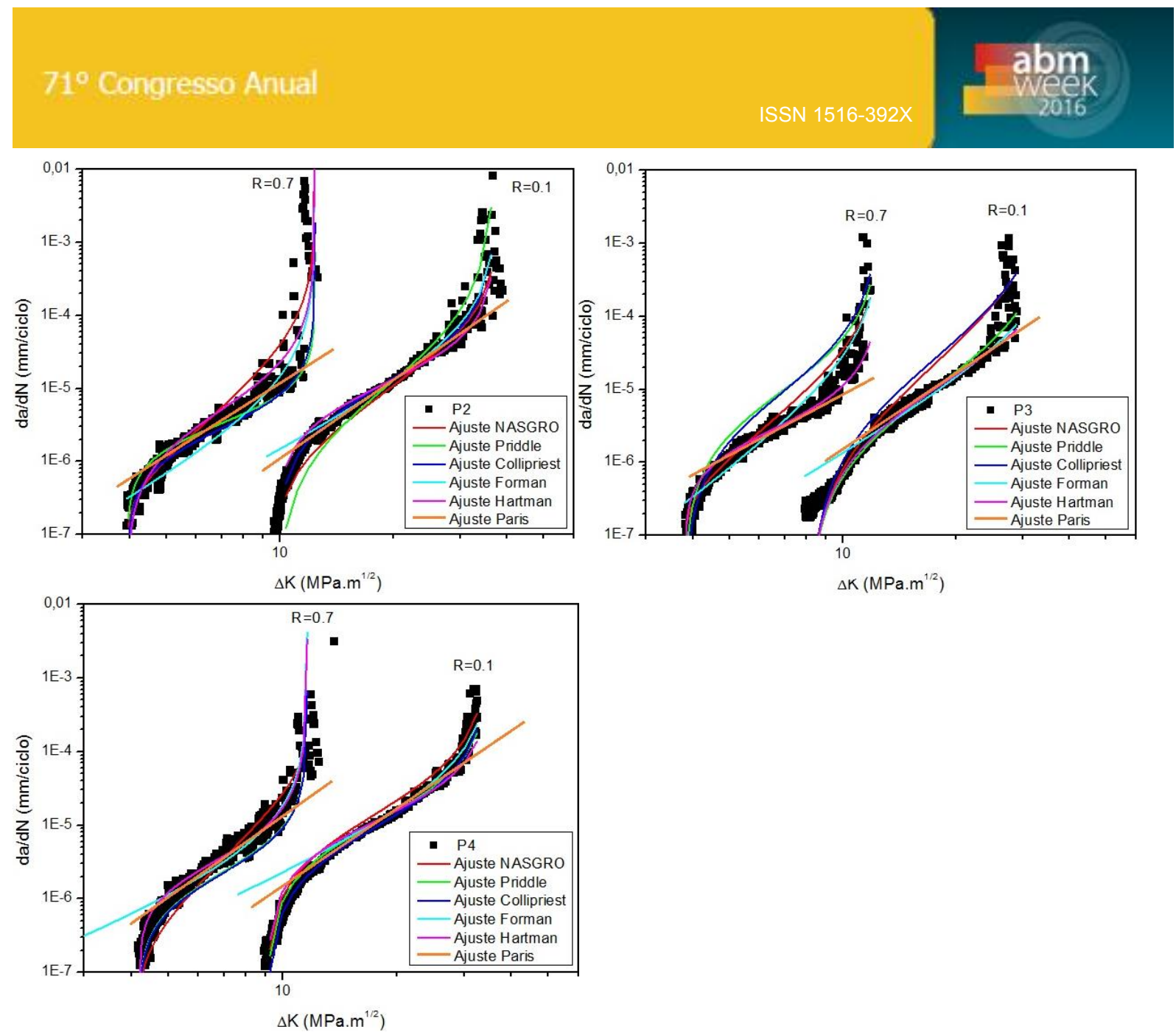

$\Delta \mathrm{K}\left(\mathrm{MPa} \cdot \mathrm{m}^{1 / 2}\right)$

Figura 6. Modelamento das curvas de propagação de trinca por fadiga dos cinco aços estudados.

A Equação de Paris leva em consideração apenas a Região II da curva, enquanto a Equação de Forman considera as Regiões II e III. As demais equações utilizadas para o modelamento levam em consideração as três regiões da curva de propagação de trinca.

Tanto para as curvas com $R=0,7$ quanto para as com $R=0,1$, o ajuste matemático de Hartman e Schijve [14], em geral, proporcionou o modelamento mais adequado.

Efetuando os cálculos para a utilização do parâmetro $K^{K}$ [15], foram encontrados para a os valores médios apresentados na Tabela 3 e "plotados" os gráficos apresentados na Figura 7. Pode-se observar que nas curvas $d a / d N \times K^{*}$ o efeito da razão $\mathrm{R}$ diminui consideravelmente, porém, para a maioria dos aços, a região próxima ao $\Delta \mathrm{K}_{\mathrm{TH}}$ está bastante dispersa.

Tabela 3 - Valores de a calculados para os cinco aços

\begin{tabular}{c|c|c|c|c|c}
\hline & P1 & P2 & P3 & P4 & S \\
\hline a médio & 0,49 & 0,55 & 0,55 & 0,50 & 0,53 \\
\hline
\end{tabular}

A pequena diferença existente entre os aços em relação à sua resistência ao crescimento de trinca por fadiga não foi suficiente para alterar o mecanismo de trincamento por fadiga entre eles. A análise fratográfica de um dos corpos de prova é apresentada na Figura 8 para a caracterização das três regiões distintas de crescimento de trinca.

Nos cinco casos há pouca diferença entre as regiões I e II de trincamento, o que ocorre devido a propagação mais rápida em ensaios com valores maiores da razão 
R. A superfície de fratura tem um aspecto "facetado" e é mais plana, caracterizando o trincamento por escorregamento de planos cristalográficos. Não foi detectada a presença de estrias nestes aços, devido ao caráter mais frágil dos materiais. $\mathrm{Na}$ região III, considerando que o material está na iminência de se fraturar, o comportamento é frágil, e o trincamento ocorre por clivagem.
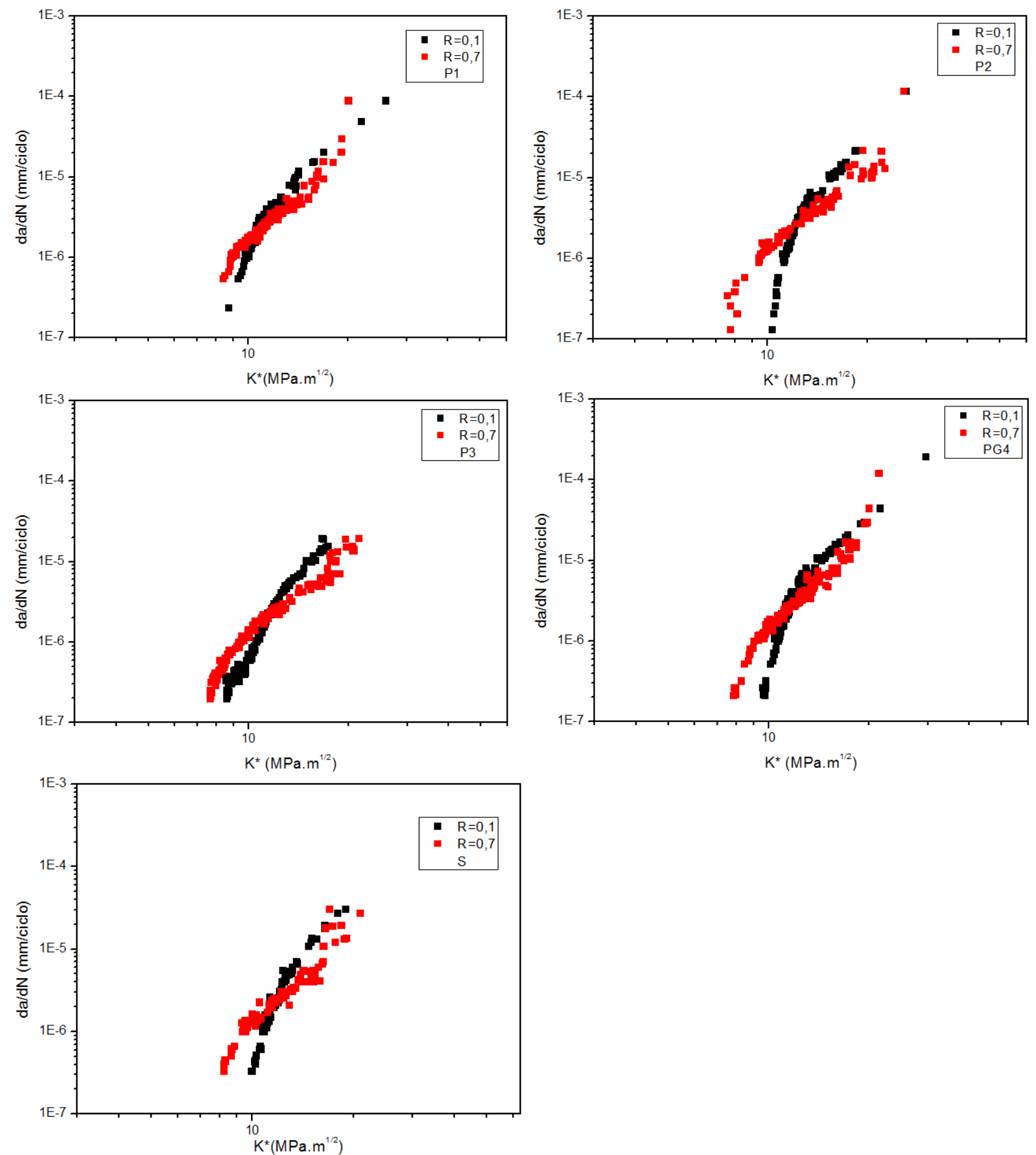

Figura 7. Curvas $d a / d N \times K^{*}$ para os cinco aços estudados. 


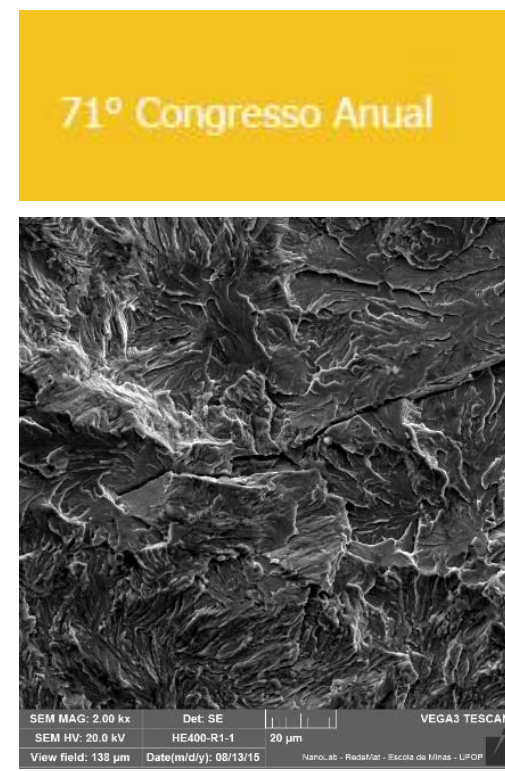

(a)

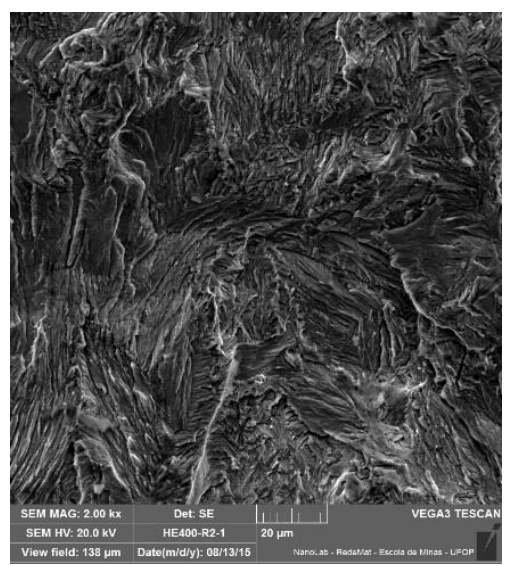

(b)

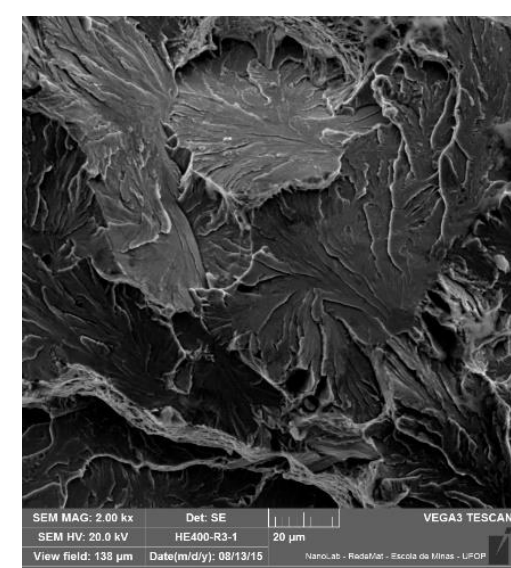

(c)

Figura 8. Superfície de fratura do corpo de prova ensaiado por fadiga com $R=0,7$, aço $P 1, M E V$, 2000X (a) região I, (b) região II e (c) região III da fratura.

\section{CONCLUSÃo}

O presente trabalho consistiu na realização de ensaios de propagação de trinca por fadiga (curvas $d a / d N \times \Delta K$ ) em cinco aços utilizados em trilhos ferroviários, comparando a influência da razão $R$ entre tensões, e no modelamento matemático das curvas obtidas para previsão do crescimento de trinca por fadiga, além da utilização de um novo parâmetro, $K^{*}$. As seguintes conclusões foram obtidas:

(1) Grande sensibilidade à razão $R$ entre tensões para os aços estudados. Fato que, somado à conclusão obtida por Moreira et al[7] de que os aços em questão possuem um valor relativamente baixo para a tenacidade à fratura, indica a necessidade de aços com maior resistência ao trincamento por fadiga para a aplicação no setor ferroviário;

(2) A equação de Hartman e Schijve [14] proporcionou o melhor ajuste às curvas sigmoidais $d a / d N \times \Delta K$;

(3) $O$ parâmetro $K^{K}$ reduziu consideravelmente o efeito da razão $R$, porém a região da curva de crescimento de trinca mais próxima ao $\Delta \mathrm{K}_{\mathrm{TH}}$ (Região I) ainda possui dispersão considerável.

\section{Agradecimentos}

À empresa VLI/FCA/VALE, pela disponibilização de materiais e corpos de prova, e bolsa de iniciação científica para T.C.G.V.

\section{REFERÊNCIAS}

1 Limberger IF. Estudo da propagação de trincas transversais por fadiga em trilhos ferroviários [tese de doutorado]. Porto Alegre: Universidade Federal do Rio Grande do Sul; 2000.

2 Silva PRT. Caracterização de trilhos ferroviários quanto à tenacidade à fratura e comportamento em fadiga [dissertação de mestrado]. Porto Alegre: Universidade Federal do Rio Grande do Sul; 1995.

3 Moreira LP, Viana T, Godefroid LB, Faria GL, Cândido LC. Study of fatigue crack growth resistance of five different steels for railroad applications. In: XIV Encontro da SBPMAT; 2015; Rio de Janeiro, Brasil. 
4 Stock R, Pipan R. RCF and wear in theory and practice - The influence of rail grade on wear and RCF. In: International Conference on Contact Mechanics and Wear of Rail / Wheel Systems; 2009; Florence, Italy. Wear; 2011. p.125-133.

5 Cannon DF, Edel KO, Grassie SL, Sawley K. Rail defects: an overview. Fat. \& Fract. of Engng. Mater. \& Struct.. 2003; 26:865-887.

6 Quadros JT. Análise de falha e caracterização metalúrgica de trilhos perlíticos [monografia]. Porto Alegre: Universidade Federal do Rio Grande do Sul; 2009.

7 Moreira LP, Viana T, Godefroid LB, Faria GL, Cândido LC. Effect of some microstructural characteristics on the fracture toughness and fatigue crack growth resistance of five different pearlitic steels for railroad application. In: 23은 International Congress of Mechanical Engineering; 2015; Rio de Janeiro, Brazil.

8 American Society for Testing and Materials. ASTM E647/08: Standard test method for measurement of fatigue crack growth rates. United States, 2008.45p.

9 Paris PC, Erdogan F. Critical analysis of crack growth propagation laws. ASME Trans. J. Basic Eng. 1963; 528-534.

10 Collipriest JE, Ehret RM, Thatcher C. Fracture mechanics equations for cyclic growth. NASA Technology Utilization Report, MFS-24447, 1973.

11 Forman RG, Mettu SR. Behavior of surface and corner cracks subjected to tensile and bending loads in Ti-6Al-4V alloy. ASTM STP. 1992; 1131:519-546.

12 Anderson TL. Fracture mechanics fundamentals and applications. Florida: Taylor and Francis Group; 2005.

13 Forman RG, Kearney VE, Engle RM. Numerical analysis of crack propagation in cyclicloaded structures. Journal of Fluids Engineering. 1967; 89(3):459-463.

14 Hartman A, Schijve J. The effects of environment and load frequency on the crack propagation law for macro fatigue crack growth in aluminium alloys. Engineering Fracture Mechanics. 1970; 1(4):615-631.

15 Kujawski D. A fatigue crack driving force parameter with load ratio effects. International Journal of Fatigue. 2001; 23:239-246.

16 El-Shabasy AB, Lewandowski JJ. Effects of load ratio, $R$, and test temperature on fatigue crack growth of fully pearlitic eutectoid steel (fatigue crack growth of pearlitic steel). International Journal of Fatigue. 2004; 26:305-309.

17 Bulloch $\mathrm{JH}$. Fatigue threshold in steels - mean stress and microstructure influences. Int. J. Pres. Ves. \& Piping. 1994; 58:103-127.

18 Masounave J, Bailon JP. The dependence of the threshold stress intensity factor on the cyclic stress ratio in fatigue of ferritic-pearlitic steels. Scripta Metallurgica. 1975; 9:723730.

19 Peixoto D, Ferreira LA. Fatigue crack propagation behavior in railway steels. International Journal of Structural Integrity. 2013; 4(4):487-500.

20 Elber W. The significance of fatigue crack closure. In: Damage Tolerance in Aircraft Structures, ASTM STP 486. Lutherville-Timonium: ASTM, 1971.

21 Godefroid LB. Fundamentos de mecânica de fratura. Ouro Preto: Imprensa Universitária da UFOP; 1995. 